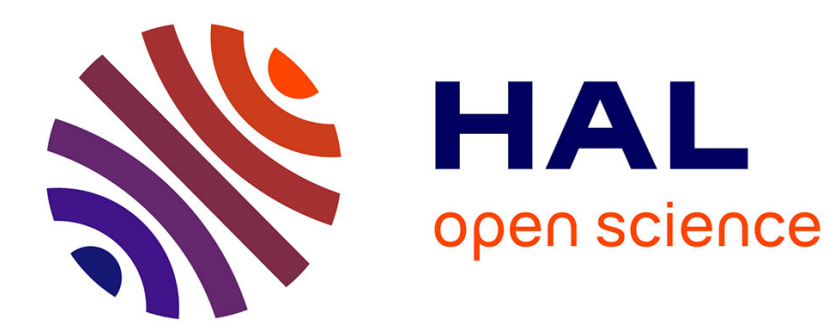

\title{
Plan d'action forestier de la forêt privée.
}

Michel de Galbert

\section{To cite this version:}

Michel de Galbert. Plan d'action forestier de la forêt privée.. Revue forestière française, 2001, 53

(3-4), pp.329-333. 10.4267/2042/5245 . hal-03442822

\section{HAL Id: hal-03442822 \\ https://hal.science/hal-03442822}

Submitted on 23 Nov 2021

HAL is a multi-disciplinary open access archive for the deposit and dissemination of scientific research documents, whether they are published or not. The documents may come from teaching and research institutions in France or abroad, or from public or private research centers.
L'archive ouverte pluridisciplinaire HAL, est destinée au dépôt et à la diffusion de documents scientifiques de niveau recherche, publiés ou non, émanant des établissements d'enseignement et de recherche français ou étrangers, des laboratoires publics ou privés. 


\section{THËIE 2. CONNAISSANCE DE LA RESSOURCE}

ET DEUELOPPEMENT INDUSTRIEL

\section{PLAN D'Action FORESTIER DE LA FORÊT PRIVÉE}

Michel de GalberT

La stratégie d'un Centre régional de la Propriété forestière (CRPF) pour le développement de la forêt privée régionale doit s'intéresser notamment à la connaissance de l'ensemble du gisement ligneux qui constitue son terrain d'action en partant du présupposé que l'ensemble de la forêt privée est concerné par l'animation du CRPF.

La connaissance du gisement de production et d'action permet de répondre à la question suivante : Pour valoriser tout le massif, quels sont les moyens à mettre en œuvre, quels sont les débouchés à trouver?

Un premier élément d'analyse du contexte général peut être fourni par la comparaison de l'accroissement et de la récolte.

Une idée du gisement peut nous être donnée par l'accroissement déterminé par l'IFN, qui le précise pour les feuillus et pour les résineux. On peut comparer cet accroissement biologique "courant" à la valorisation qui en est faite par l'exploitation forestière.

L'enquête annuelle de branche, corrigée par les avis d'experts sur les exploitations en forêt privée, et les enquêtes complémentaires sur le bois de feu nous donnent les prélèvements recensés. Pour le bois de feu, on a réparti les prélèvements régionaux précis pour approcher les chiffres départementaux en appliquant un prorata des surfaces de taillis feuillus des départements (cf. tableau I, ci-dessous). En outre, l'IFN peut aussi fournir des indications sur la mortalité et les prélèvements annuels moyens.

Il faut noter que les enquêtes de consommation de bois de feu issus de forêts de l'ADEME sont très précises au niveau régional.

TABLEAU I

Région Rhône-Alpes : comparaison accroissement/récolte

\begin{tabular}{|c|c|c|c|c|c|c|c|}
\hline & $\begin{array}{l}\text { Accrois- } \\
\text { sement } \\
\text { Forêt } \\
\text { privée } \\
\text { (m³/an) }\end{array}$ & $\begin{array}{l}\text { Récolte } \\
\text { Forêt } \\
\text { privée } \\
\text { (m³/an) }\end{array}$ & $\begin{array}{l}\text { Récolte } \\
\text { de bois } \\
\text { d'œuvre } \\
\text { Forêt } \\
\text { privée } \\
\text { (m³/an) }\end{array}$ & $\begin{array}{c}\text { Récolte } \\
\text { de bois } \\
\text { d'industrie } \\
\text { Forêt } \\
\text { privée } \\
\text { (m³/an) }\end{array}$ & $\begin{array}{l}\text { Estimation } \\
\text { auto- } \\
\text { consom- } \\
\text { mation de } \\
\text { bois de feu } \\
\text { (m3/an) }\end{array}$ & $\begin{array}{c}\text { Total } \\
\text { estimé } \\
\text { récolte } \\
\text { Forêt } \\
\text { privée } \\
\text { (m³/an) }\end{array}$ & $\begin{array}{l}\text { Récolte / } \\
\text { accrois- } \\
\text { sement } \\
(\%)\end{array}$ \\
\hline Feuillus .... & 3078200 & 227000 & 178900 & 48100 & 1500000 & 1727000 & 56,10 \\
\hline Résineux ...... & 3545800 & 1122100 & 993400 & 128700 & 0 & 1122100 & 31,65 \\
\hline Total ........ & 6624000 & 1349100 & 1172300 & 176800 & 1500000 & 2849100 & 43,01 \\
\hline
\end{tabular}

Sources: IFN-SERFOB, 1999. 


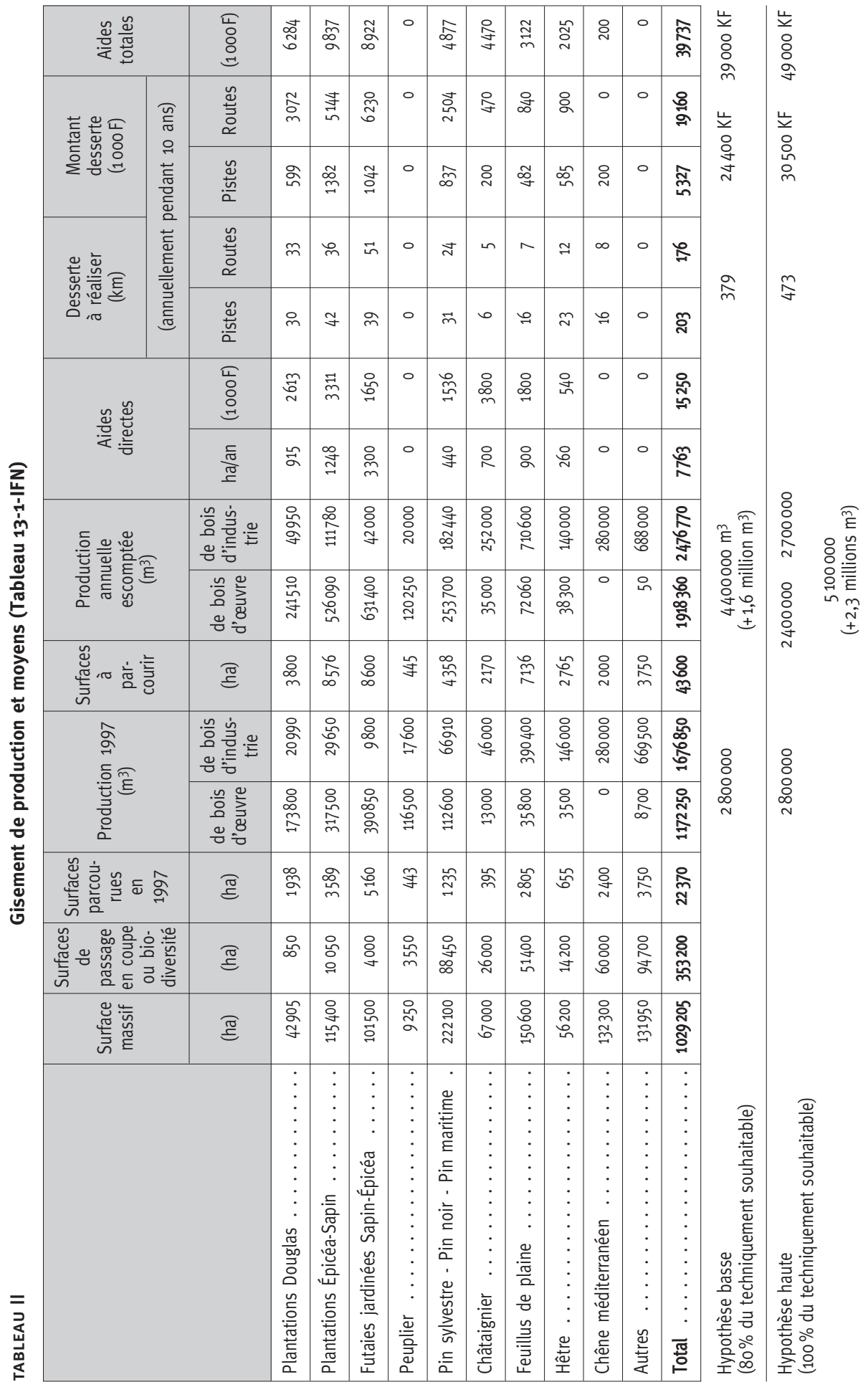


Au total, on constate un prélèvement moyen de $43 \%$, sans doute inférieur à cause du décalage de date inventaire/enquête annuelle de branche. Ce prélèvement est-il techniquement normal en Rhône-Alpes, où la forêt privée comprend de vieux peuplements de sapinières dans la Loire et dans les Alpes, ainsi que de jeunes peuplements en pleine croissance ? Dans une forêt équilibrée, l'addition "accroissement plus passage à la futaie" égale théoriquement la somme "récolte plus mortalité”.

\section{COMMENT CONNAÎTRE LE PRÉLÈVEMENT OPTIMAL ?}

Il s'avère nécessaire de faire un travail plus technique à partir des peuplements forestiers, d'itinéraires sylvicoles simples et de la connaissance que les techniciens du CRPF ont du terrain, soit une sorte d'aménagement régional qui ferait fi des contraintes de dessertes et de morcellement dans un premier temps.

Cela revient à calculer une ressource techniquement mobilisable.

\section{Utilisation des données de l'IFN}

On se sert tout d'abord du tableau des types de peuplements et des surfaces, volumes, accroissement, production brute, mortalité correspondante (tableau 13-1, publication IFN - cf. tableau II, p. 330).

Pour les sapinières irrégulières ou en voie de régularisation, il n’y a pas de difficultés à définir un prélèvement-type homogène sur l'ensemble des surfaces du type "sapinières", mais ceci est insuffisant pour les reboisements. Il convient de se reporter au tableau des essences résineuses réparties par classes d'âges en les décalant dans le temps pour obtenir les surfaces actuelles (tableau 8-2, publication IFN).

\section{Itinéraire sylvicole}

On applique la sylviculture optimale préconisée par le CRPF (tableau 2-3 Loire, publication IFN).

\section{Exemple :}

Pour le Douglas, on applique les trois types d'intervention : première éclaircie $=40 \mathrm{~m}^{3}$, éclaircies de rattrapage $=70 \mathrm{~m}^{3}$ et amélioration des peuplements de Douglas $=60 \mathrm{~m}^{3}$, et l'on décompose ces prélèvements en bois d'industrie et bois d'œuvre.

\section{Application et surfaces estimées}

On estime que l'on doit pouvoir prélever $80 \%$ de la surface optimale, ce qui représente un prélèvement sur $8 \%$ du territoire par an pour un prélèvement tous les 10 ans et évidemment davantage pour un prélèvement tous les 6 ans par exemple. On estime un prélèvement bois d'œuvre / bois d'industrie et les hectares à parcourir et l'on estime les dessertes correspondantes à réaliser, par application d'un ratio de desserte $/ \mathrm{km}^{2}$ (tableau III, p. 332).

\section{Comparaison des objectifs et des moyens actuels}

En appliquant les moyens actuels de mobilisation à l'hectare ou en kilomètre, on calcule l'ensemble des aides directes à mettre en place, le montant de la desserte, les aides sylvicoles, le reboisement, l'élagage, etc., les moyens d'animation du CRPF et de la coopération ainsi que les emplois potentiels dans la filière sans compter les retours de TVA générés (synthèse stratégique). 


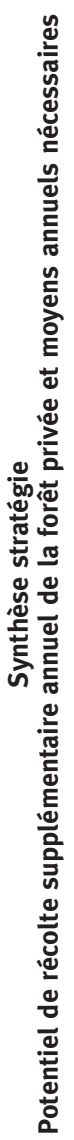

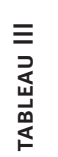

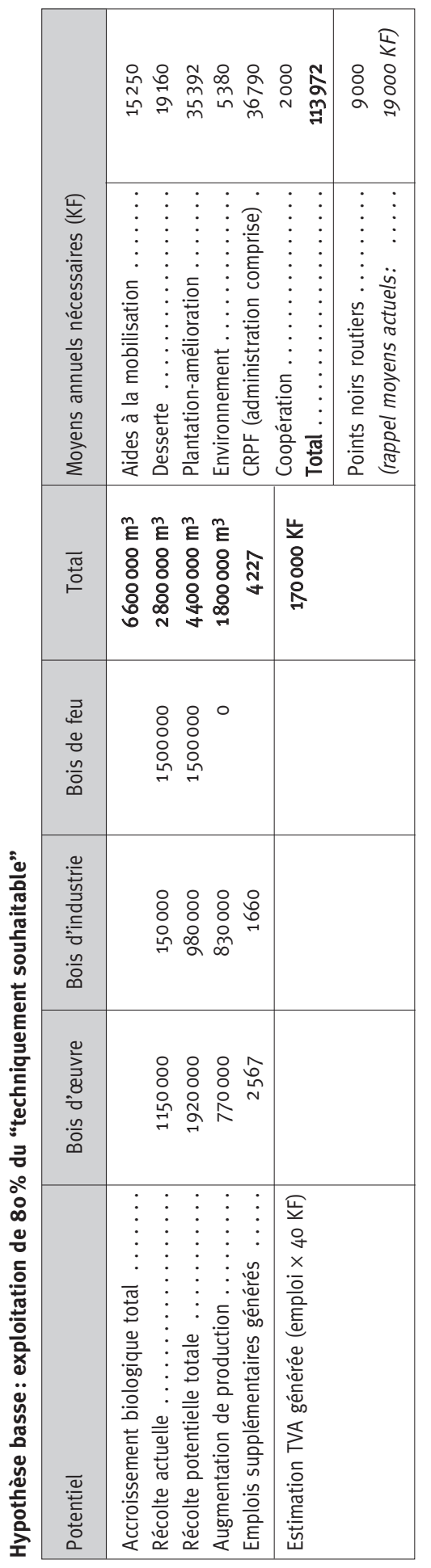

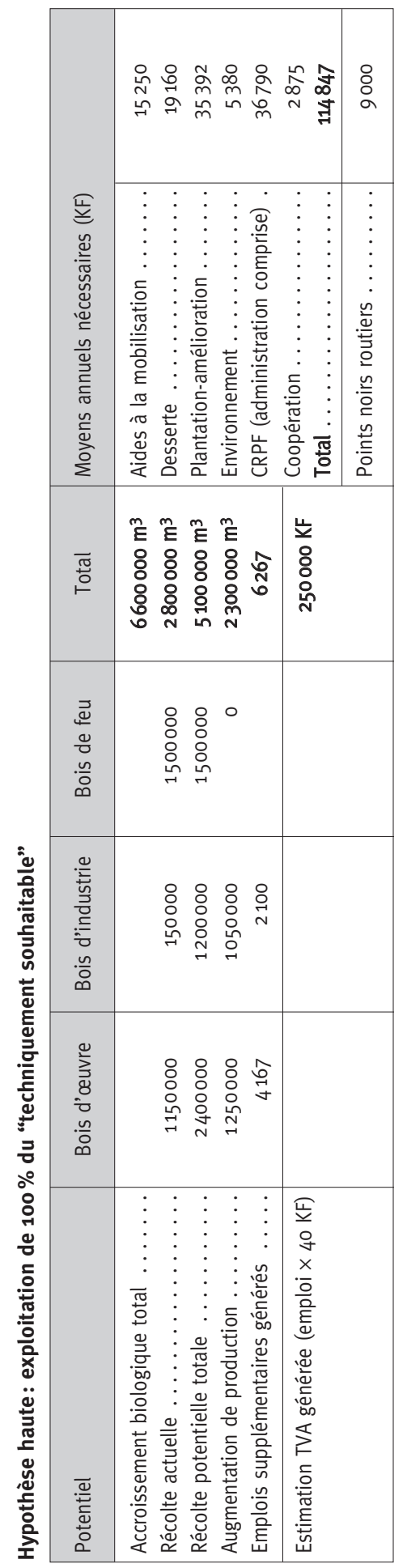


Ces investissements et charges pourraient être sensiblement allégés par l'amélioration des débouchés comme ceux de la trituration, par certains outils issus des nouveaux textes en préparation, ainsi que par les moyens de communication et d'animation qui permettent également de développer les structures associatives et la participation des propriétaires eux-mêmes dans le cadre des structures précitées.

Moyennant ces avancées, on constate que les crédits d'investissement et d'animation en forêt privée ont des retombées économiques et sociales intéressantes et rapides.

\section{CONCLUSIONS}

On le voit, l'utilisation de l'IFN est le socle indispensable à la connaissance du gisement forestier et de son développement. Par ailleurs, si l'on considère que l'accroissement de la région Rhône-Alpes a doublé en 25 ans, il est clair que l'actualisation des données de l'IFN doit permettre de s'adapter à ces évolutions très importantes en une génération. Le passage de l'Inventaire tous les 10 ans est donc un rythme indispensable.

Il serait intéressant de reprendre cette simulation pour les 20 et 30 ans à venir et avec des options simples mais claires comme la poursuite des reboisements (30 000 ha/an, 10000 ha/an), arrêt de la régénération naturelle par non maîtrise des populations de gibier, etc.

Michel de GALBERT

Directeur du CENTRE RÉGIONAL

DE LA PROPRIÉTÉ FORESTIÈRE RHÔNE-ALPES

Parc de Crécy

18, avenue du Général-de-Gaulle

F-69771 SAINT-DIDIER-AU-MONT-D’OR CEDEX

(crpf-rhone-alpes@wanadoo.fr) 